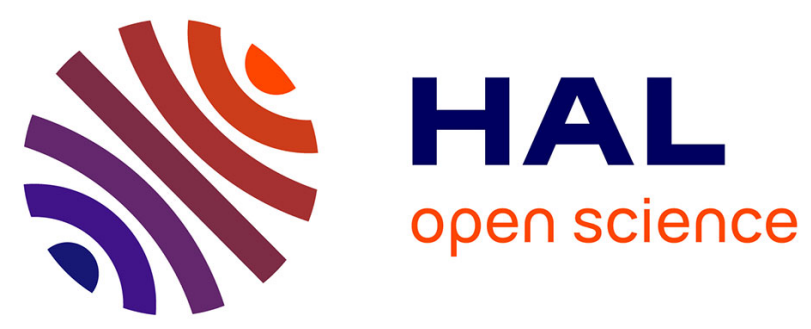

\title{
Etude comparative de l'expérience émotionnelle subjective chez des adolescents présentant une symptomatologie dépressive associée ou non à des conduites à risques
}

Vincent Bréjard, Aurélie Pasquier, Agnes Bonnet, Jean-Louis Pedinielli

\section{To cite this version:}

Vincent Bréjard, Aurélie Pasquier, Agnes Bonnet, Jean-Louis Pedinielli. Etude comparative de l'expérience émotionnelle subjective chez des adolescents présentant une symptomatologie dépressive associée ou non à des conduites à risques . L'Encéphale, 2011, 37 (4), pp.257-265. 10.1016/j.encep.2010.12.003 . hal-01273489

\section{HAL Id: hal-01273489 \\ https://hal-amu.archives-ouvertes.fr/hal-01273489}

Submitted on 12 Feb 2016

HAL is a multi-disciplinary open access archive for the deposit and dissemination of scientific research documents, whether they are published or not. The documents may come from teaching and research institutions in France or abroad, or from public or private research centers.
L'archive ouverte pluridisciplinaire HAL, est destinée au dépôt et à la diffusion de documents scientifiques de niveau recherche, publiés ou non, émanant des établissements d'enseignement et de recherche français ou étrangers, des laboratoires publics ou privés. 
Etude comparative de l'expérience émotionnelle subjective chez des adolescents présentant une symptomatologie dépressive associée ou non à des conduites à risques.

Comparative study of the subjective emotional experience among adolescents showing depressive symptoms associated or not with risk-taking behaviors

\author{
V. Bréjard ${ }^{\mathrm{a}^{\mathrm{k}}}$, A. Pasquier ${ }^{\mathrm{b}}$, A. Bonnet ${ }^{\mathrm{c}}, \mathrm{J}-\mathrm{L}$ Pedinielli ${ }^{\mathrm{c}}$
}

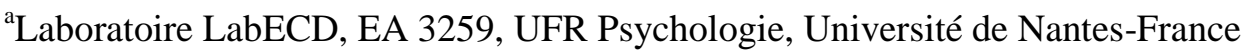

${ }^{\mathrm{b}}$ Laboratoire PsyCLE, EA 3273, IUFM d'Aix-Marseille, Université de Provence-France

${ }^{\mathrm{c}}$ Laboratoire LPCP, EA 3278, UFR Psychologie-Sciences de l'éducation, Université de Provence-

France

*Auteur correspondant : UFR de Psychologie Chemin de la Censive du Tertre BP 81227 44312 NANTES Cedex 3 France. tél: 0240141076 e-mail: vincent.brejard@univ-nantes.fr 


\section{Résumé}

Prise de risque et dépression à l'adolescence constituent un problème pour le psychopathologue, au sens où leurs relations peuvent être interprétées de différentes manières : comportements signant l'échec de la lutte contre la dépression ou encore dépression secondaire voire «réactionnelle» aux conséquences négatives de ceux-ci, ou simple association. Un certain nombre de travaux ont souligné le rôle des émotions et de leurs dysfonctionnements comme facteurs associés ou explicatifs de celle-ci. L'objectif de cette étude était de caractériser les relations entretenues par ces deux troubles en utilisant une approche comparative. A cette fin, à partir d'un échantillon de 488 adolescents, 4 groupes ont été constitués : deux groupes monosymptomatiques présentant soit des conduites à risques, soit une symptomatologie dépressive, un groupe présentant une association des deux troubles, et un groupe contrôle sans dépression ni conduites à risques avérées. Les participants ont complété une série d'autoévaluations de la dépression (CES-D), des conduites à risques (YRBSS), du niveau de conscience émotionnelle (LEAS), et de l'intensité émotionnelle subjective (DES). Les résultats obtenus permettent de caractériser chaque groupe par une articulation spécifique du niveau de conscience émotionnelle et de l'intensité émotionnelle subjective, particulièrement dans le cas des émotions négatives. Ces résultats peuvent être interprétés comme liés à un arrêt développemental chez les adolescents à différents niveaux de conscience émotionnelle amenant à des dysfonctionnements spécifiques dans les capacités à utiliser l'information émotionnelle afin de s'adapter à l'environnement.

Mots clés: Adolescence-Conscience émotionnelle-conduites à risques-dépression-comorbidité 
Emotion awareness and subjective emotional intensity : specificity in risk-taking and depression in adolescence

Introduction . Relationships between risk-taking behaviors and depressive disorders in youth are considered as a complex psychopathological problematic. Previous findings showed strong correlations between substance abuse, risk-taking and depressive symptoms. But the question remains open of the potential common factors that depression and risk-taking may have. Besides researches focusing on personality dimensions, some others highlight the potential role played by emotions and their pathological functioning. In these studies, pathological emotional processing such as alexithymia or specific lack of emotional intensity was linked to both risk-taking behaviors and depressive disorders. The aim of this study was to investigate potential specific emotional profiles of adolescents engaged in pathological risk-taking or depressive symptomatology, versus adolescents presenting an association of the two. Method. 488 adolescents $\left(\mathrm{m}_{\mathrm{age}}=14,93 S D=1,44\right)$ with 257 boys $\left(\mathrm{m}_{\mathrm{age}}=15, S D=1,51\right)$ and 231 girls $\left(\mathrm{m}_{\mathrm{age}}=14,52 S D=1,23\right)$ were spread in four groups. First group was of adolescents engaged in high level risk-taking, second was that of adolescents showing both high risktaking and high depressive symptoms, third was composed of only depressed adolescents and the fourth group was the control group with no pathological expression. The four groups completed a set of three inventories: the Youth Risk Behavior Surveillance Scale (YRBSS), the Level of Emotional Awareness Scale (LEAS) and the Differential Emotiona Scale (DES). Results. . Adolescents engaged in risk-taking have the lower level of emotional awareness and subjective emotional intensity, while adolescents of the second group (depression with risk-taking) have higher level on both measures. Depressed adolescents present the higher score of emotional awareness of the pathological groups, but lower than controls. However, their level of emotional awareness of others and their subjective negative emotional intensity were higher than the control group. Discussion. The results may be explained by a specific developmental hypothesis, in which emotional awareness growth from early period of life to late childhood, allowing children to gradually experiment more and more complex subjective emotional experience. In other words, emotions may have an important role in introducing a latency time between environment stimuli and oriented responses. In case of early developmental arrest, there may be a lack of emotion information, because of poverty in emotional awareness, leading to a pathological emotional processing. Clinical implications of this interpretation are discussed.

Keywords: adolescence- emotional awareness- depression-risk-taking-comorbidity 


\section{Introduction}

L'adolescence est une période du développement riche en changements physiques et psychologiques. Ces profonds remaniements créent une vulnérabilité à présenter des troubles psychopathologiques, parmi lesquels la dépression et les comportements à risques représentent deux tableaux fréquemment rencontrés. Pour un certain nombre d'adolescents, on retrouve une comorbidité entre symptomatologie dépressive et expression comportementale telle que l'abus de substances, la prise de risques ou encore l'agressivité. D'un point de vue clinique, les conduites de mise en danger sont fréquemment associées aux troubles de l'humeur, comme le soulignent plusieurs études [25, 34, 41]. Plus précisément, la dépression serait en cause dans l'engagement dans des conduites à risques, notamment dans l'abus de substances ou la sexualité à risque [2,23]. Cependant, ces données ne permettent pas de préciser si le trouble dépressif était antérieur aux conduites à risques, et s'il est survenu parallèlement ou secondairement. Dans l'exemple de la dépendance tabagique, les adolescents fumeurs ont deux fois plus de risques de présenter un trouble dépressif sur un an, alors qu'un trouble dépressif constitue inversement un facteur de vulnérabilité à la consommation de substances [5]. Cette association entre dépression et consommation de substances pourrait s'expliquer par le fait qu'elles partagent des facteurs de risques, tels que les antécédents de consommation de produits dans la fratrie par exemple, ou encore des facteurs psychosociaux tels que des troubles internalisés (anxiété-dépression dans l'enfance, sentiment de rejet vis-àvis des pairs...) ou externalisés (instabilité comportementale, troubles de la concentration, et agressivité). Les premiers sont supposés favoriser préférentiellement une dépression à l'adolescence, tandis que les seconds seraient plus volontiers liés à la consommation de substances. De plus, on retrouve dans 36 à $80 \%$ des cas selon les études, une comorbidité entre symptômes dépressifs et comportements tels que école buissonnière, vol, fugue, violence verbale et comportementale [15]. Parmi les différents facteurs de vulnérabilité à présenter des troubles dépressifs et des conduites à risques à l'adolescence, le rôle des émotions et plus particulièrement celui de l'éprouvé subjectif et de l'intensité de celui-ci ont été soulignés.

\section{Emotion et troubles psychopathologiques : dépression et expression comportementale}

Certains dysfonctionnements émotionnels ont été retrouvés dans les pathologies dépressives et les addictions. Plus précisément, les relations de l'alexithymie (littéralement «absence de mots pour décrire les émotions » [38]) avec ces deux groupes de troubles ont fait l'objet de 
nombreux travaux Ainsi, les sujets alexithymiques présenteraient une tendance à ressentir les émotions avec une intensité inférieure à la normale, tout en présentant une humeur dépressive accentuée [21, 31]. Dans le champ des conduites pathologiques, telles que les addictions, l'alexithymie a fait l'objet de nombreuses contributions. ]. L'association entre alexithymie et trouble de l'humeur a été régulièrement retrouvée dans les conduites addictives et d'abus de substances. Un fonctionnement alexithymique est ainsi lié à la dépression chez une proportion significative de patients présentant une dépendance à l'alcool [19]. Alexithymie et dépression sont également observés de manière concomitante dans la dépendance au tabac [24] ainsi qu'aux substances psychoactives hors alcool [30, 40].

Dans cette perspective, et afin de formuler une hypothèse étiologique de différents troubles émotionnels (notamment alexithymie et anhédonie), Lane et Schwartz ont mis l'accent sur la représentation -consciente- de l'expérience émotionnelle en se référant aux conceptions développementales issues des travaux de Piaget et de Werner [27, 28]. Pour eux, la conscience émotionnelle se développerait progressivement d'une manière similaire au développement intellectuel, passant par une succession de niveaux hiérarchiques allant du plus simple au plus élaboré, en cinq phases. La conscience émotionnelle serait à l'origine (stade «sensorimoteur réflexe ») la perception d'un niveau plus ou moins élevé d'activation physiologique, de sensations indifférenciées. Au second stade («sensorimoteur») les éprouvés encore peu discriminés conduiraient essentiellement à des tendances à l'action. Au troisième niveau («préopératoire ») seules les émotions «basiques » seraient identifiées et discriminées. Le niveau suivant («opératoire concret») verrait l'avènement d'émotions différenciées, alors que le cinquième et dernier niveau (« opératoire formel ») correspondrait à la capacité à expérimenter et reconnaître des états émotionnels complexes et différenciés, tant chez soi-même que chez autrui. Cette conception permet ainsi d'expliquer certains déficits émotionnels par des arrêts développementaux à des niveaux où l'expérience émotionnelle ne fait l'objet que d'une faible différenciation, notamment dans le cas de l'alexithymie ou du coping répressif comme le proposent Lane et al. [29]. Le concept de conscience émotionnelle a montré son intérêt dans différentes études portant sur des entités cliniques variées telles que les troubles dépressifs, anxieux $[13,32]$ les troubles des conduites alimentaires $[9,18]$, ou encore la dépendance à l'alcool [4] et la polytoxicomanie [22]. La conscience émotionnelle a également fait l'objet d'investigations dans les troubles internalisés chez l'enfant, où un faible niveau de conscience émotionnelle a été observé [36] ainsi que chez l'adolescent où des capacités limitées dans le traitement de l'information émotionnelle ont été mises en évidence chez les adolescents engagés dans des prises de risques répétées [20]. 
L'expérience émotionnelle est également impliquée dans la dépression et les conduites à risques à travers l'intensité émotionnelle ressentie, et plus spécifiquement dans le cas des émotions négatives. Les adolescents déprimés ont tendance à expérimenter des émotions négatives de manière plus fréquente et plus intense que les sujets non déprimés [16]. De plus, dans une étude portant sur 152 adolescents, Silks \& Morris ont mis en évidence que des stratégies de régulation émotionnelle peu efficaces pour réguler les émotions négatives (de type déni ou rumination) étaient significativement liées à des niveaux élevés de dépression et de comportements problématiques [39].

Suite à ces différents éléments issus de travaux antérieurs, nous avons souhaité caractériser les adolescents présentant des conduites à risques sans thymie dépressive, des conduites à risques avec thymie dépressive, et une thymie dépressive sans conduites à risques, sur le plan du niveau de conscience émotionnelle et de l'intensité de l'expérience émotionnelle subjective.

A notre sens, les conduites à risques seraient déterminées par un faible niveau de conscience émotionnelle associé à une faible intensité émotionnelle ressentie, alors que la dépression serait plutôt liée à une faible conscience émotionnelle - quoique supérieure à celle des sujets preneurs de risque- et à une forte intensité émotionnelle négative.

Notre hypothèse est qu'il existe, à l'échelle de niveaux de conscience émotionnelle et à l'évaluation de l'intensité émotionnelle, des différences significatives entre les trois typologies symptomatiques précitées. Ces différences seront ordonnées de la manière suivante du niveau le plus faible au plus élevé : adolescents preneurs de risques sans symptômes dépressifs, adolescents présentant une comorbidité conduites à risques/symptômes dépressifs, adolescents dépressifs, et adolescents témoin (sans troubles avérés) aura les niveaux de conscience émotionnelle et d'intensité émotionnelle les plus élevés, tandis que les adolescents présentant les deux symptomatologies et celui constitué d'adolescents dépressifs auront une position intermédiaire, les sujets du groupe présentant uniquement un trouble dépressif obtenant toutefois des scores supérieurs aux adolescents avec pathologie mixte comportementale/dépressive.

\section{Méthode}

\section{Participants}

Nous avons constitué un échantillon de 488 adolescents (âge moyen $=14,93 \pm 1,44$ ) à partir d'établissements du secondaire (classes de $5^{\mathrm{e}}$ à $3^{\mathrm{e}}$ de collège, de filières $4^{\mathrm{e}}$ et $3^{\mathrm{e}}$ SEGPA et de 2 de à terminale de lycée d'enseignement général et de lycée professionnels). Les adolescents 
ont été rencontrés par l'expérimentateur ou ses collaborateurs en classe et leur accord écrit, ainsi que celui de leurs parents et du chef d'établissement, a été demandé après explication des objectifs de l'étude, en précisant qu'à tout moment ils pouvaient se retirer de l'étude. 565 adolescents ont été contactés, et seuls 488 ont retourné des questionnaires exploitables $(86,37 \%)$. Ainsi 257 garçons (âge moyen $=15,38 \pm 1,51$ ) et 231 filles (âge moyen $=14,52 \pm$ 1, 23) ont participé à l'étude. Le recueil des données s'est déroulé de Septembre 2003 à Juin 2004. Les passations se déroulaient collectivement en notre présence ou celle de deux collaborateurs.

\section{Evaluations}

L'évaluation des conduites à risques a été réalisée avec la forme abrégée de l'échelle d'évaluation des risques chez les jeunes (Youth Risk Behavior Surveillance Scale) [1]. Il s'agit d'une échelle d'auto-évaluation en dix items à choix forcé de type vrai/faux. Il n'existe à ce jour pas de traduction française de cet instrument, ce qui nous a amené à effectuer la traduction en double-sens : anglais-français et français-anglais. 10 items ont été ajoutés afin d'étendre l'évaluation au cadre scolaire. Les qualités psychométriques de l'outil se sont révélées satisfaisantes (alpha de Cronbach=.71).

La présence d'un état dépressif a été évalué avec la CES-D (échelle de dépression du Centre d'études épidémiologiques) [35]. Elle a été traduite en français [17] et ses propriétés psychométriques ont été étudiées avec un échantillon d'adolescents [11].

La conscience émotionnelle a été mesurée par la LEAS ou échelle de niveau de conscience émotionnelle. Elle est composée de 20 items décrivant une scène amenant le sujet à s'imaginer en interaction avec une autre personne, dans le cadre d'une situation issue du quotidien. Le sujet doit répondre réaliser deux descriptions portant sur l'état émotionnel ressenti : l'une commençant la phrase par « je me sentirais ou je ressentirai.... » et l'autre par «l'autre personne ressentirait ou se sentirait....». Deux sous-échelles permettent ainsi d'obtenir une évaluation des capacités du sujet à être conscient de ses états émotionnels (échelle Soi), ainsi que de ses capacités à les conceptualiser chez autrui (échelle Autrui). Les réponses émotionnelles sont cotées avec une grille comportant une liste de termes émotionnels et une note s'y référent. Les réponses sont cotées de 0 à 5 et permettent d'obtenir un score général compris entre 0 et 100 . Les qualités psychométriques de l'instrument apparaissent satisfaisantes [10, 26]. Notre échantillon étant composé d'adolescents, nous avons utilisé une forme adaptée de l'échelle, déjà utilisée précédemment. [7]. Dans cette 
forme modifiée, la consistance interne pour cette étude est congruente avec celle obtenue dans la forme initiale ( $\alpha$ de Cronbach $=.83$ ) [26].

Afin de disposer d'une comparaison fiable entre les expérimentateurs, $25 \%$ du protocole a fait l'objet d'une double cotation, ce qui a permis de calculer une corrélation entre les deux séries de .92 , semblable aux travaux francophones déjà publiés.

L'intensité de l'éprouvé subjectif a été évaluée avec l'échelle d'émotions différentielle d'Izard afin d'évaluer la qualité et l'intensité de l'éprouvé subjectif en auto-évaluation. Elle est constituée de 40 items correspondant à dix émotions fondamentales. Elle a été traduite et validée en français par Ouss et al.[33]. L’échelle a été complétée deux fois : une fois après une description écrite d'un évènement positif puis une seconde après celle d'un évènement négatif. Deux scores pour chaque évènement ont été calculés par additions des scores aux émotions négatives et aux émotions positives.

\section{Procédure}

Nous avons constitué quatre groupes en croisant l'évaluation des conduites à risques (codée NC si inférieures à la médiane de 7 ou présence codée C) avec celle de la dépression (absence codée ND ou présence codée $\mathrm{D}$, avec un seuil de 26 [11]). Ces quatre groupes ont été codés comme suit: adolescents présentant des conduites à risques sans trouble dépressif (CND), adolescents présentant des conduites à risques et un trouble dépressif (CD), adolescents ne présentant pas de conduites à risques mais un trouble dépressif (NCD), adolescents sans troubles comportementaux ni dépressif (NCND).

Afin de valider notre hypothèse, nous avons procédé à une analyse multiple de variance (MANOVA) avec test post-hoc LSD. La variable indépendante était le type de groupe et les variables dépendantes correspondaient aux niveaux de conscience émotionnelle (soi, autrui, total), et d'intensité émotionnelle (émotions positives, négatives, cas d'un évènement positif et négatif). L’âge a été entré comme covariable et le sexe a été contrôlé. Les traitements statistiques ont été réalisés avec le logiciel SPSS 11.5.

\section{Résultats}

Une partie des statistiques descriptives a déjà été présentée ailleurs (Bréjard, Bonnet \& Pedinielli, en révision). Cependant, une autre partie ainsi que les analyses multivariées présentées ici sont originales. 


\section{Analyses descriptives}

On observe (tableau 1) que les adolescents présentent un niveau supérieur de conduites à risques $(\mathrm{t}=2, \mathrm{p}=0.05)$, tandis que les adolescentes présentent un niveau supérieur de dépression $(\mathrm{t}=-2,61, \mathrm{p}=0,01)$. De même, les filles ont un niveau de conscience émotionnel supérieur aux garçons $(\mathrm{t}=-3,51, \mathrm{p}>0.001)$, et déclarent ressentir les émotions positives (cas d'un évènement positif) et négatives (cas d'un évènement négatif) de manière plus intense.

Insérer le tableau 1 ici

\section{Différences intergroupes}

Pour toutes les analyses, le sexe et l'âge ont été contrôlés, et n'ont pas produit d'effets significatifs, sauf mentionnés dans le texte

\section{Niveau de conscience émotionnelle}

Les résultats sont présentés dans le tableau 2. Nous avons analysé l'effet du type de groupe sur le niveau de conscience émotionnelle global, et avons pu observer qu'il était significatif $(\mathrm{F}(3,479)=84,83 ; \mathrm{p}<.0001)$. Quand on examine les différences intergroupes, on constate que les adolescents du groupe CND ont le niveau de conscience émotionnelle le plus bas $\left(m_{\mathrm{CND}}=37,25 \pm 3,24\right)$. Les adolescents du groupe mixte, noté $\mathrm{CD}$, obtiennent un score supérieur $\left(\mathrm{m}_{\mathrm{CD}}=45,45 \pm 4,44\right)$ au groupe $\mathrm{CND}$, mais inférieur aux adolescents des deux autres groupes. Le groupe des adolescents NCD a quant à lui un score supérieur aux deux groupes d'adolescents présentant des conduites à risques $\left(\mathrm{m}_{\mathrm{NCD}}=47,07 \pm 4,27\right)$. Le groupe témoin a le niveau de conscience émotionnelle le plus élevé $\left(\mathrm{m}_{\mathrm{NCD}}=50,76 \pm 5,94\right)$.

Nous avons également analysé les sous-scores à l'échelle de niveau de conscience émotionnelle. Il existe un effet du sexe $(\mathrm{F}(1,479=4,87 ; \mathrm{p}=.027)$. Mais cet effet n'est pas dépendant de la variable groupe, pour laquelle on observe un effet principal $(\mathrm{F}(3,479)=48,84$; $\mathrm{p}<.0001)$. Les résultats montrent néanmoins que cet effet n'est pas retrouvé dans toutes les différences intergroupes. En effet, si le groupe $\mathrm{CND}$ a un score moyen $\left(\mathrm{m}_{\mathrm{CND}}=33,48 \pm 3,28\right)$ significativement inférieur à ceux des autres groupes, on n'observe en revanche pas de différence significative entre les groupes $C D\left(m_{C D}=41,03 \pm 4,55\right)$ et $\mathrm{NCD}\left(\mathrm{m}_{\mathrm{NCD}}=42,18 \pm 4,61\right)$. Le groupe témoin NCND a, quant à lui, le niveau de conscience émotionnelle intrasubjectif le plus élevé $\left(\mathrm{m}_{\mathrm{NCND}}=45,08 \pm 6,81\right)$. Concernant le NCE intersubjectif, on peut observer qu'il y a un effet du type de groupe $(\mathrm{F}(3,479)=64,27 ; \mathrm{p}<.0001)$, où les adolescents du groupe CND ont un score significativement inférieur $\left(\mathrm{m}_{\mathrm{CND}}=28,23 \pm 3,58\right)$ aux autres adolescents. Les adolescents du groupe CD ont un score supérieur aux adolescents CND sur le plan du niveau 
de conscience émotionnelle intersubjectif $\left(\mathrm{m}_{\mathrm{CD}}=35,05 \pm 6,29\right)$, mais inférieur à ceux des groupes $\mathrm{NCD}\left(\mathrm{m}_{\mathrm{NCD}}=42,13 \pm 4,32\right)$ et $\mathrm{NCND}\left(\mathrm{m}_{\mathrm{NCND}}=40,51 \pm 7,17\right)$. Nous pouvons en outre observer que, de manière surprenante, les adolescents déprimés sans conduites à risques ont un score supérieur aux adolescents du groupe témoin.

Insérer le tableau 2 ici

\section{Intensité émotionnelle ressentie dans le cas de la confrontation à un évènement positif}

Les différences intergroupes au score d'l'intensité émotionnelle sont présentées dans le tableau 3. Concernant l'intensité des émotions positives, il existe un effet significatif du type de groupe $(F(3,479)=24,83 ; \mathrm{p}<.0001)$. Le groupe des adolescents CND ressent les émotions de manière moins intense $\left(\mathrm{m}_{\mathrm{CND}}=5,80 \pm 1,59\right)$ que les autres groupes $\left(\mathrm{m}_{\mathrm{CD}}=9,48 \pm 3,55\right.$; $\left.\mathrm{m}_{\mathrm{NCD}}=9,57 \pm 3,33 ; \quad \mathrm{m}_{\mathrm{NCND}}=10,10 \pm 2,37\right)$. Les résultats obtenus au score d'intensité émotionnelle négative montrent qu'il existe un effet principal du type de groupe $(\mathrm{F}(3,479)=13,15 ; \mathrm{p}=.0001)$. Plus précisément on constate une absence de différence significative entre d'une part les groupes CND $\left(\mathrm{m}_{\mathrm{CND}}=4,58 \pm 6,46\right)$ et $\mathrm{NCND}$ $\left(\mathrm{m}_{\mathrm{NCND}}=4,53 \pm 7,34\right)$ et d'autre part les groupes $\mathrm{CD} \quad\left(\mathrm{m}_{\mathrm{CD}}=9,75 \pm 11,63\right)$ et $\mathrm{NCD}$ $\left(\mathrm{m}_{\mathrm{NCD}}=9,02 \pm 10,47\right)$. Les adolescents présentant une symptomatologie dépressive ont tendance à ressentir un haut niveau d'émotions négatives face à un évènement positif.

\section{Intensité émotionnelle ressentie dans le cas de la confrontation à un évènement négatif}

Les adolescents preneurs de risque sans état dépressif éprouvent les émotions positives de manière moins intense $\left(\mathrm{m}_{\mathrm{CND}}=0,25 \pm 0,58\right)$ que les autres groupes $\left(\mathrm{m}_{\mathrm{CD}}=1,50 \pm 1,94 ; \mathrm{p}=.015\right.$ $\left.\mathrm{m}_{\mathrm{NCD}}=1,53 \pm 2,95 ; \mathrm{p}=.006-\mathrm{m}_{\mathrm{NCND}}=1,22 \pm 2,82 ; \mathrm{p}=.032\right)$. Au niveau des différences intragroupes (en fonction du sexe), on n'observe qu'une différence significative, au sein du groupe présentant un état dépressif : les filles $\left(\mathrm{m}_{\mathrm{F}}=1,19 \pm 2,58\right)$ ont un niveau d'émotions positives inférieur aux garçons $\left(\mathrm{m}_{\mathrm{G}}=1,97 \pm 3,34, \mathrm{p}=.016\right)$. Dans le cas des émotions négatives, il existe également un effet principal du type de groupe $(\mathrm{F}(3,479)=84,36 ; \mathrm{p}<.0001)$. Les adolescents du groupe CND sont ceux qui ressentent les émotions négatives de la manière la moins intense $\left(\mathrm{m}_{\mathrm{CND}}=17,12 \pm 4,37\right)$. Ils ont un niveau inférieur aux groupes $\mathrm{CD}$ $\left(\mathrm{m}_{\mathrm{CD}}=37,30 \pm 7,08 ; \mathrm{p}<.0001\right), \quad \mathrm{NCD} \quad\left(\mathrm{m}_{\mathrm{NCD}}=40,17 \pm 8,67 ; \quad \mathrm{p}<.0001\right), \quad$ et $\mathrm{NCND}$ $\left(\mathrm{m}_{\mathrm{NCND}}=31,32 \pm 9,28 ; \mathrm{p}<.0001\right)$. Les adolescents du groupe $\mathrm{CD}$ ont, quant à eux, un score supérieur au groupe témoin $(\mathrm{p}<.0001)$, mais inférieur au groupe NCD ( $\mathrm{p}=.016)$. Le groupe NCD a, pour sa part, le score le plus élevé.

Insérer le tableau 3 ici 


\section{Discussion}

Nous avions posé l'hypothèse que le niveau de conscience émotionnelle du groupe « conduites à risques » serait le plus faible des quatre groupes. Cette hypothèse est validée. Concernant l'échelle intrasubjective, les mêmes résultats sont observés, à l'exception d'une absence de différence entre les deux groupes d'adolescents présentant un état dépressif. Cela ne valide que partiellement notre hypothèse. Nous pouvons penser que les capacités cognitivo-émotionnelles déterminent le niveau de dépression, mais ne permettent pas de discriminer les deux groupes sur le plan de la présence ou de l'absence de conduites à risques. Nos résultats montrent également que si le groupe CND (conduites à risques sans symptômes dépressifs) est celui dont le niveau de conscience émotionnel intersubjectif est le plus faible, suivi du groupe $\mathrm{CD}$ (conduites à risques avec symptômes dépressif), le groupe ayant le niveau le plus élevé est celui d'adolescents présentant uniquement des symptômes dépressifs, et non le groupe témoin. Nous pensons qu'il est possible d'interpréter ce résultat comme indicatif de la tendance excessive, non pas à une focalisation sur ses propres états émotionnels, mais plutôt à la préoccupation exagérée pour ceux d'autrui.

Nous observons également que les émotions négatives et positives sont ressenties de manière différenciée par chaque groupe. Le groupe CD ressent les émotions de manière plus intense que le groupe CND, mais cependant moins intensément que les adolescents uniquement déprimés et les témoins. Nous observons que l'intensité émotionnelle ressentie par les adolescents du groupe NCD est inférieure à celle du groupe témoin (NCND). Nous nous attendions également à ce qu'ils aient une intensité émotionnelle supérieure aux deux autres groupes (CND et CD). Or si les adolescents présentant un état dépressif ont bien une intensité émotionnelle supérieure aux groupes CND et CD, elle l'est également chez ceux du groupe témoin, ne validant pas notre hypothèse concernant ce groupe. Les adolescents sans conduites à risques excessive ni symptomatologie dépressive présentent donc à la fois un niveau de conscience plus élevé et une intensité émotionnelle moins forte que les adolescents présentant des symptômes dépressifs. Ceci pourrait être considéré comme indicatif de l'existence chez les adolescents NCD d'une hypersensibilité émotionnelle, elle-même déterminée par un trouble de la régulation des émotions. Ce trouble pourrait consister en une incapacité à moduler l'intensité des émotions ressenties, et conduirait à un débordement possible par des éprouvés subjectifs négatifs intenses générateurs de dépression. Chez certains adolescents, l'externalisation par le recours à des comportements pathologiques pourrait constituer une 
solution défensive comme le suggèrent certaines conceptions des relations entre comportements (pathologiques) et troubles thymiques. Celles-ci considèrent les comportements comme «l'expression du trouble de l'humeur au travers d'une autre symptomatologie»[12]. Ils ont une fonction économique, entrant dans le cadre d'une stratégie défensive de type «anti-pensée » et «anti-représentation ».

$\mathrm{Si}$ avec Scherer on suppose un rôle de régulation adaptative aux émotions, permettant à l'individu d'introduire un «temps de latence » entre l'évaluation de l'environnement et la réponse, comportementale ou autre [37], les adolescents qui présentent des conduites à risques auraient, du fait de leur faible niveau de conscience émotionnelle, un dysfonctionnement émotionnel caractérisé par un mécanisme d'atténuation portant spécifiquement sur les émotions négatives. Ce dysfonctionnement pourrait selon nous avoir des conséquences majeures en ne permettant plus au sujet, du fait d'une faible conscience de ses propres états subjectifs, d'utiliser les émotions pour réguler ses comportements. Cette interprétation pourrait être étayée par le caractère volontiers réactionnel des prises de risques et des réactions comportementales de certains adolescents et par les difficultés dont ils témoignent à en expliciter les déterminants.

A un niveau plus global, nous pensons que ce déterminisme pourrait être lui-même sous-tendu par un arrêt développemental à un stade où les aptitudes à identifier ses états internes sont encore immatures, et ne permettent pas le recours à l'information émotionnelle pour réguler les comportements. Cet arrêt développemental pourrait être liés à des contextes environnementaux (traumatismes, psychopathologie parentale). En effet, même si pour le moment des données empiriques mettant directement en relation trouble du développement cognitivo-émotionnel et environnement précoce ne sont pas disponibles, la présence de problèmes comportementaux et psychopathologiques chez les adolescents est souvent liée à des histoires personnelles faites d'instabilité et d'insécurité environnementale [6, 8, 14].

Toutefois, ces résultats doivent être considérés en tenant compte de certaines limites possibles. Au regard de l'autoévaluation utilisée, nous ne pouvons exclure l'influence de la désirabilité sociale dans cette procédure Toutefois, l'utilisation de la LEAS permet de limiter ce biais, tout au moins pour l'aspect conscience émotionnelle [9]. Dans le cadre de développements ultérieurs, l'utilisation conjointe d'une méthodologie quantitative et qualitative, notamment par l'utilisation d'entretiens, pourrait utilement contrôler ce biais [3]. Une seconde limite concerne les écarts d'effectifs entre les groupes. Cette différence d'effectifs a pu induire des biais dans les tests statistiques, malgré l'utilisation d'une procédure spécifique d'analyse de la variance pour échantillons de tailles inégales (somme 
des carrés type III). Enfin, l'absence de contrôle de la psychopathologie générale pourrait expliquer une part non négligeable de la variabilité dans nos résultats ; elle aurait, en outre, permis d'analyser les comorbidités potentielles, notamment avec les troubles anxieux.

\section{Conclusion}

Les relations entre expressions comportementales à l'adolescence et dépression sont complexes. Ce travail apporte des éléments complémentaires sur le rôle de la conscience émotionnelle et de l'intensité émotionnelle subjective, en permettant de spécifier les caractéristiques propres à chaque type d'expression symptomatique. Les résultats obtenus soulèvent la question du lien possible entre conduites à risques comme exacerbation de comportements exploratoires insuffisamment régulés par les émotions, et symptomatologie dépressive comme échec du même processus régulateur des émotions, avec une information émotionnelle dans un cas insuffisante, et dans un autre excessive conduisant à des symptômes dépressifs. Les adolescents présentant un tableau mixte seraient quant à eux confrontés à une problématique spécifique où les prises de risques constitueraient une tentative de lutte contre des éprouvés subjectifs négatifs impossible à traiter et verbaliser du fait d'un trop faible niveau de conscience émotionnelle. Des études à venir devront permettre de confirmer ces hypothèses par une méthodologie longitudinale, amenant à analyser dans quelle mesure l'apparition de signes dépressifs pourraient conduire à l'engagement dans des conduites à risques, ou au contraire à les limiter. D'un point de vue clinique, ces résultats soutiennent la pertinence d'une prise en charge avec un étayage sur les sensations et la motricité alliée à des temps d'élaboration de ces éprouvés, qui pourra favoriser la complexification et la construction de représentations conscientes des émotions. Enfin, ce type de travail pourrait permettre de transformer des émotions-traumas ne pouvant faire l'objet d'une représentation psychique en émotions-informations susceptibles d'être travaillées et utilisées.

Conflits d'intérêts : aucun

\section{Références}

[1] Aklin WM, Lejuez CW, Zvolensky MJ, Kahler CW, Gwadz M (2005) Evaluation of behavioral measures of risk taking propensity with inner city adolescents. Behaviour Research and Therapy 43:215-228

[2] Bancroft J, Janssen E, Carnes L, Goodrich D, Strong D, Long JS (2004) Sexual activity and risk taking in young heterosexual men: the relevance of sexual arousability, mood, and sensation seeking. J Sex Res 41:181-192

[3] Blanchet A (1997) Recherches sur le langage en psychologie clinique. Dunod, Paris 
[4] Bochand L, Nandrino JL (Sous presse) Niveaux de conscience émotionnelle chez les sujets alcoolodépendants et abstinents. L'Encéphale

[5] Bonomo Y, Coffey C, Wolfe R, Lynskey M, Bowes G, Patton G (2001) Adverse outcomes of alcohol use in adolescents. Addiction 96:1485-1496

[6] Borowsky IW, Resnick MD (1998) Environmental stressors and emotional status of adolescents who have been in special education classes. Arch Pediatr Adolesc Med 152:377382

[7] Bréjard V, Bonnet A, Pedinielli JL (2005) Développement cognitivo-émotionnel, régulation des émotions et comportements à risques : une étude exploratoire chez l'adolescent. Neuropsychiatr Enfance Adolesc 53:395-400

[8] Broidy LM, Nagin DS, Tremblay RE, Bates JE, Brame B, Dodge KA, Fergusson D, Horwood JL, Loeber R, Laird R, Lynam DR, Moffitt TE, Pettit GS, Vitaro F (2003) Developmental trajectories of childhood disruptive behaviors and adolescent delinquency: a six-site, crossnational study. Dev Psychol 39:222-245

[9] Bydlowski S, Corcos M, Jeammet P, Paterniti S, Berthoz S, Laurier C, Chambry J, Consoli SM (2005) Emotion-processing deficits in eating disorders. Int J Eat Disord 37:321-329

[10] Bydlowski S, Corcos M, Paterniti S, Guilbaud O, Jeammet P, Consoli SM (2002) Validation de la version francaise de l'echelle des niveaux de conscience emotionnelle. Encephale 28:310-320

[11] Chabrol H, Montovany A, Chouicha K, Duconge E (2002) Etude de la CES-D dans un échantillon de 1,953 adolescents scolarisés. Encephale 28:429-432

[12] Corcos M, Jeammet P (2005) Les dépressions à l'adolescence. Dunod, Paris

[13] Donges US, Kersting A, Dannlowski U, Lalee-Mentzel J, Arolt V, Suslow T (2005) Reduced awareness of others' emotions in unipolar depressed patients. J Nerv Ment Dis 193:331-337

[14] Fergusson DM, Horwood LJ, Lynskey M (1994) The childhoods of multiple problem adolescents: a 15-year longitudinal study. J Child Psychol Psychiatry 35:1123-1140

[15] Ferro T, Carlson GAn, Grayson P, Klein DN (1994) Depressive disorders:dinstinctions in children. J Am Acad Child Adoles Psychiatry 33:664-670

[16] Forbes EE, Williamson DE, Ryan ND, Dahl RE (2004) Positive and negative affect in depression: influence of sex and puberty. Ann N Y Acad Sci 1021:341-347

[17. Fuhrer T, Rouillon F (1989) La version française de l'échelle CES-D (Center for Epidemiologic Studies-Depression Scale). Description et traduction de l'échelle d'autoévaluation. Psychiatr Psychobiologie 4:163-166

[18] Gilboa-Schechtman E, Avnon L, Zubery E, Jeczmien P (2006) Emotional processing in eating disorders: specific impairment or general distress related deficiency? Depress Anxiety 23:331339

[19] Guilbaud O, Loas G, Corcos M, Speranza M, Stephan P, Perez-Diaz F, Venisse JL, Guelfi JD, Bizouard P, Lang F (2002) L'alexithymie dans les conduites de dependance et chez le sujet sain : valeur en population francaise et francophone: Alexithymia in addictive behaviors and in healthy subjects: results of a study in French speaking subjects. Ann Med Psychol 160:7785

[20] Hessler DM, Katz LF Brief report: Associations between emotional competence and adolescent risky behavior. J of Adolescence 33:241-246

[21] Honkalampi K, Hintikka J, Tanskanen A, Lehtonen J, Viinamaki H (2000) Depression is strongly associated with alexithymia in the general population. J Psychosom Research 48:99104

[22] Jouanne C, Edel Y, Carton S (2005) Déficits émotionnels chez des patients polytoxicomanes. Ann Med Psychol 163:625-630

[23] King CA, Ghaziuddin N, McGovern L, Brand E, Hill E, Naylor M (1996) Predictors of comorbid alcohol and substance abuse in depressed adolescents. J Am Acad Child Adolesc Psychiatry 35:743-751

[24] Kojima M, Furukawa T, Nagaya T, Tokudome S (2003) Smoking, depression and alexithymia. J Psychosom Research 55:156 
[25] Kosunen E, Kaltiala-Heino R, Rimpela M, Laippala P (2003) Risk-taking sexual behaviour and self-reported depression in middle adolescence--a school-based survey. Child Care Health Dev 29:337-344

[26] Lane RD, Quinlan DM, Schwartz GE, Walker PA, Zeitlin SB (1990) The Levels of Emotional Awareness Scale: a cognitive-developmental measure of emotion. J Pers Assess 55:124-134

[27] Lane RD, Schwartz GE (1987) Levels of emotional awareness: a cognitive-developmental theory and its application to psychopathology. Am J Psychiatry 144:133-143

[28] Lane RD, Schwartz GE (1990) The neuropsychophysiology of emotion. Funct Neurol 5:263266

[29] Lane RD, Sechrest L, Riedel R, Shapiro DE, Kaszniak AW (2000) Pervasive emotion recognition deficit common to alexithymia and the repressive coping style. Psychosom Med 62:492-501

[30] Liappas J, Peppas E, Rabavilas AD, Paparrigopoulos T, Papavasiliou P, Chrisitodoulou GN (1998) The relationship between alexithymia and depression-like symptomatology in drug users. Eur Neuropsychopharmacology 8:5-14

[31] Lumley MA, Downey K, Stettner L, Wehmer F, Pomerleau OF (1994) Alexithymia and negative affect: relationship to cigarette smoking, nicotine dependence, and smoking cessation. Psychother Psychosom 61:156-162

[32] Novick-Kline P, Turk CL, Mennin DS, Hoyt EA, Gallagher CL (2005) Level of emotional awareness as a differentiating variable between individuals with and without generalized anxiety disorder. J Anxiety Disord 19:557-572

[33] Ouss L, Carton S, Jouvent R, Widlocher D (1990) Traduction et validation de l'echelle d'emotions differentielles d'Izard. Exploration de la qualification verbale des emotions. Encephale 16:453-458

[34] Pesa JA, Cowdery JE, Westerfield RC, Wang M (1997) Self-reported depression and risktaking behaviors among Hispanic adolescents. Psychol Rep 81:235-243

[35] Radloff LS (1977) The CES-D scale: a self-report depression scale for research in the general population. Applied Psychological Measurements 1:385-401

[36] Rieffe C, Oosterveld P, Miers AC, Meerum Terwogt M, Ly V (2008) Emotion awareness and internalising symptoms in children and adolescents: The Emotion Awareness Questionnaire revised. Pers Ind Differences 45:756-761

[37] Scherer KR (2000) Emotions as episodes of subsystems synchronization driven by nonlinear appraisal processes. In: Lewis MD, Granic I (eds) Emotion, development, and selforganization: Dynamic systems approaches to emotional development. Cambridge University Press, New York, NY, p 70-99

[38] Sifneos PE (1973) The prevalence of "alexithymic" characteristics in psychosomatic patients. Psychoth Psychosomatics Vol. 22:255-262

[39] Silk JS, Steinberg L, Morris AS (2003) Adolescents' emotion regulation in daily life: links to depressive symptoms and problem behavior. Child Dev 74:1869-1880

[40] Speranza M, Corcos M, Stéphan P, Loas G, Pérez-Diaz F, Lang F, Venisse JL, Bizouard P, Flament M, Halfon O, Jeammet P (2004) Alexithymia, Depressive Experiences, and Dependency in Addictive Disorders. Subst Use Misuse 39:551-579

[41] Whalen CK, Jamner LD, Henker B, Delfino RJ (2001) Smoking and moods in adolescents with depressive and aggressive dispositions: evidence from surveys and electronic diaries. Health Psychol 20:99-111 
Tableau 1. Statistiques desciptives et différences intersexes aux variables de l'étude

\begin{tabular}{|c|c|c|c|c|c|c|c|c|}
\hline & \multicolumn{2}{|l|}{ Total } & \multicolumn{2}{|l|}{$\mathrm{F}$} & \multicolumn{2}{|l|}{$\mathrm{M}$} & \multirow[b]{2}{*}{$\mathrm{t}(2,486)$} & \multirow[b]{2}{*}{$\mathrm{p}$} \\
\hline & M & SD & M & SD & M & SD & & \\
\hline Conduites à risques & 2,26 & $(2,33)$ & 2,06 & $(2,28)$ & 2,48 & $(2,38)$ & 2,00 & 0,05 \\
\hline Dépression & 18,7 & $(10,20)$ & 19,84 & $(10,96)$ & 17,44 & $(9,14)$ & $-2,61$ & 0,01 \\
\hline NCE Soi & 42,62 & $(6,47)$ & 43,57 & $(6,67)$ & 41,57 & $(6,09)$ & $-3,45$ & $<0,001$ \\
\hline NCE Autrui & 39,14 & $(7,23)$ & 40,15 & $(7,07)$ & 38,02 & $(7,26)$ & $-3,27$ & 0,00 \\
\hline NCE Général & 47,72 & $(6,30)$ & 48,65 & $(6,44)$ & 46,67 & $(5,99)$ & $-3,51$ & $<0,001$ \\
\hline $\begin{array}{l}\text { IE+ situation } \\
\text { positive }\end{array}$ & 9,5 & $(3,06)$ & 9,79 & $(2,82)$ & 9,17 & $(3,28)$ & $-2,25$ & 0,03 \\
\hline IE- situation positive & 7,72 & $(9,55)$ & 8,39 & $(9,69)$ & 6,97 & $(9,35)$ & $-1,64$ & 0,10 \\
\hline $\begin{array}{l}\text { positive } \\
\text { IE+ situation }\end{array}$ & 9,53 & $(4,81)$ & 9,74 & $(4,59)$ & 9,31 & $(5,04)$ & $-0,98$ & 0,33 \\
\hline $\begin{array}{l}\text { négative } \\
\text { IE- situation }\end{array}$ & 1,29 & $(2,77)$ & 1,23 & $(2,75)$ & 1,35 & $(2,79)$ & 0,48 & 0,63 \\
\hline $\begin{array}{l}\text { négative } \\
\text { AE situation }\end{array}$ & 33,76 & $(10,55)$ & 34,81 & $(10,47)$ & 32,59 & $(10,54)$ & $-2,34$ & 0,02 \\
\hline négative & 7,73 & $(4,19)$ & 7,44 & $(4,09)$ & 8,05 & $(4,28)$ & 1,59 & 0,11 \\
\hline
\end{tabular}

NCE: Niveau de Conscience Emotionnelle; IE+: Intensité Emotionnelle émotions positives; IE-: Intensité Emotionnelle émotions négatives; AE: Activation Emotionnelle 
Tableau 2. Comparaisons multiples intergroupes (Manova avec tests post-hocs LSD) au niveau de conscience émotionnelle

\begin{tabular}{|c|c|c|c|c|c|c|}
\hline Variable dépendante & Groupe 1 & Groupe 2 & (G1-G2) & $p$ & $95 \%$-IC inf & $\begin{array}{c}95 \%-\mathrm{IC} \\
\text { sup }\end{array}$ \\
\hline \multirow[t]{6}{*}{ NCE Soi } & \multirow[t]{3}{*}{ CND } & $\mathrm{CD}$ & $-6,83$ & 0,00 & $-9,14$ & $-4,52$ \\
\hline & & NCD & $-13,9$ & 0,00 & $-16,01$ & $-11,79$ \\
\hline & & NCND & $-12,29$ & 0,00 & $-14,32$ & $-10,26$ \\
\hline & \multirow[t]{2}{*}{$\mathrm{CD}$} & NCD & $-7,08$ & 0,00 & $-8,75$ & $-5,40$ \\
\hline & & NCND & $-5,46$ & 0,00 & $-7,03$ & $-3,89$ \\
\hline & NCD & NCND & 1,62 & 0,01 & 0,36 & 2,87 \\
\hline \multirow[t]{6}{*}{ NCE Autrui } & \multirow[t]{3}{*}{ CND } & $\mathrm{CD}$ & $-7,55$ & 0,00 & $-9,70$ & $-5,40$ \\
\hline & & NCD & $-8,7$ & 0,00 & $-10,66$ & $-6,74$ \\
\hline & & NCND & $-11,6$ & 0,00 & $-13,49$ & $-9,72$ \\
\hline & \multirow[t]{2}{*}{$\mathrm{CD}$} & $\overline{N C D}$ & $-1,15$ & 0,15 & $-2,70$ & 0,40 \\
\hline & & NCND & $-4,05$ & 0,00 & $-5,51$ & $-2,59$ \\
\hline & NCD & NCND & $-2,9$ & 0,00 & $-4,07$ & $-1,74$ \\
\hline \multirow[t]{6}{*}{ NCE Général } & \multirow[t]{3}{*}{ CND } & $\mathrm{CD}$ & $-8,2$ & 0,00 & $-10,11$ & $-6,28$ \\
\hline & & NCD & $-9,82$ & 0,00 & $-11,57$ & $-8,07$ \\
\hline & & NCND & $-13,51$ & 0,00 & $-15,19$ & $-11,83$ \\
\hline & \multirow[t]{2}{*}{$\mathrm{CD}$} & $\mathrm{NCD}$ & $-1,63$ & 0,02 & $-3,01$ & $-0,24$ \\
\hline & & NCND & $-5,31$ & 0,00 & $-6,61$ & $-4,01$ \\
\hline & NCD & NCND & $-3,69$ & 0,00 & $-4,73$ & $-2,64$ \\
\hline
\end{tabular}

*p<.05. NCE: Niveau de Conscience Emotionnelle 
Tableau 3 Comparaisons multiples intergroupes (Manova avec tests post-hocs LSD) à l'intensité émotionnelle

\begin{tabular}{|c|c|c|c|c|c|c|}
\hline Variable dépendante & Groupe 1 & Groupe 2 & (G1-G2) & $p$ & $95 \%-\mathrm{IC}$ inf & $95 \%$-IC sup \\
\hline \multirow[t]{6}{*}{ IE+ situation positive } & \multirow[t]{3}{*}{ CND } & $\mathrm{CD}$ & $-3,69$ & 0,00 & $-4,78$ & $-2,59$ \\
\hline & & $\mathrm{NCD}$ & $-3,78$ & 0,00 & $-4,78$ & $-2,78$ \\
\hline & & NCND & $-4,3$ & 0,00 & $-5,26$ & $-3,34$ \\
\hline & \multirow[t]{2}{*}{$\mathrm{CD}$} & NCD & $-0,09$ & 0,82 & $-0,88$ & 0,70 \\
\hline & & NCND & $-0,62$ & 0,10 & $-1,36$ & 0,13 \\
\hline & NCD & NCND & $-0,52$ & 0,08 & $-1,12$ & 0,07 \\
\hline \multirow[t]{6}{*}{ IE- situation positive } & \multirow[t]{3}{*}{ CND } & $\mathrm{CD}$ & $-5,38$ & 0,00 & $-9,00$ & $-1,75$ \\
\hline & & NCD & $-4,65$ & 0,01 & $-7,97$ & $-1,34$ \\
\hline & & NCND & $-2,4$ & 0,14 & $-5,59$ & 0,78 \\
\hline & \multirow[t]{2}{*}{$\mathrm{CD}$} & NCD & 0,72 & 0,59 & $-1,90$ & 3,35 \\
\hline & & NCND & 2,97 & 0,02 & 0,51 & 5,44 \\
\hline & NCD & NCND & 2,25 & 0,03 & 0,28 & 4,22 \\
\hline \multirow[t]{6}{*}{ IE+ situation négative } & \multirow[t]{3}{*}{ CND } & $\mathrm{CD}$ & $-1,3$ & 0,02 & $-2,36$ & $-0,24$ \\
\hline & & NCD & $-1,28$ & 0,01 & $-2,25$ & $-0,31$ \\
\hline & & NCND & $-0,97$ & 0,04 & $-1,90$ & $-0,04$ \\
\hline & \multirow[t]{2}{*}{$\mathrm{CD}$} & NCD & 0,02 & 0,96 & $-0,74$ & 0,79 \\
\hline & & NCND & 0,33 & 0,37 & $-0,39$ & 1,05 \\
\hline & NCD & NCND & 0,31 & 0,29 & $-0,27$ & 0,88 \\
\hline \multirow[t]{6}{*}{ IE- situation négative } & \multirow[t]{3}{*}{ CND } & $\mathrm{CD}$ & $-20,18$ & 0,00 & $-23,43$ & $-16,93$ \\
\hline & & NCD & $-23,05$ & 0,00 & $-26,02$ & $-20,08$ \\
\hline & & NCND & $-14,2$ & 0,00 & $-17,06$ & $-11,35$ \\
\hline & \multirow[t]{2}{*}{$\mathrm{CD}$} & $\mathrm{NCD}$ & $-2,87$ & 0,02 & $-5,23$ & $-0,52$ \\
\hline & & NCND & 5,97 & 0,00 & 3,77 & 8,18 \\
\hline & NCD & NCND & 8,85 & 0,00 & 7,08 & 10,61 \\
\hline
\end{tabular}

*p<.05. IE+: Intensité Emotionnelle émotions positives; IE-: Intensité Emotionnelle émotions négatives 